\title{
Relation Between Benchmark Dose and No-Observed-Adverse-Effect Level in Clinical Research: Effects of Daily Alcohol Intake on Blood Pressure in Japanese Salesmen
}

\author{
Miwako Dakeishi, ${ }^{1}$ Katsuyuki Murata, ${ }^{1 *}$ Akiko Tamura, ${ }^{1}$ and Toyoto Iwata ${ }^{1}$
}

\begin{abstract}
The benchmark dose (BMD) is defined as the dose that corresponds to a specific change in an adverse response compared to the response in unexposed subjects, and the lower $95 \%$ confidence limit is termed the benchmark dose level (BMDL). In this study, the threshold of daily ethanol intake affecting blood pressure was calculated by both the BMD approach and multiple logistic regression analysis to clarify the relation between the BMDL and noobserved-adverse-effect level (NOAEL). Systolic and diastolic blood pressures (SBP and DBP) and daily ethanol intake were explored in 1,100 Japanese salesmen. The SBP and DBP were positively related to daily ethanol intake $(p<0.001)$ when adjusting for possible confounders such as age, body mass index, and smoking status. The adjusted risk for hypertension ( $\mathrm{SBP} \geq 140 \mathrm{mmHg}$ or $\mathrm{DBP} \geq 90 \mathrm{mmHg}$ ) increased significantly when daily ethanol intake exceeded $60 \mathrm{~g} / \mathrm{day}$, and the categorical dose of interest was 60.1-90 g/day. The BMDL and BMD of ethanol intake for increased SBP and DBP were estimated to be approximately 60 and $75 \mathrm{~g} /$ day, respectively. These findings suggest that the BMDL and BMD correspond to the NOAEL and lowest-observed-adverse-effect level, respectively, if the sample number of clinical data is large enough to confirm the dose-response association.
\end{abstract}

KEY WORDS: Benchmark dose calculation; blood pressure; clinical research; daily ethanol intake; no-observed-adverse-effect level

\section{INTRODUCTION}

For risk management of a hazardous substance in the environment, it is necessary to identify the discernible threshold of the substance affecting human health. Previously, the threshold was obtained from the lowest level producing the adverse effect (e.g., the lowest average value among exposed popu-

\footnotetext{
1 Department of Environmental Health Sciences, Akita University School of Medicine, Akita, Japan.

* Address correspondence to Katsuyuki Murata, Department of Environmental Health Sciences, Akita University School of Medicine, 1-1-1 Hondo, Akita 010-8543, Japan; tel: +81-18-8846085; fax: +81-18-836-2608; winestem@med.akita-u.ac.jp.
}

lations with specific dysfunction), termed the lowestobserved-adverse-effect level (LOAEL). ${ }^{(1,2)}$ With respect to the estimation of such thresholds, the more desirable method has been described to use the highest reported dose or exposure level for which no toxicity was observed, i.e., the no-observed-adverse-effect level (NOAEL). As an alternative to the NOAEL or LOAEL approach, however, benchmark dose (BMD) calculations have been applied in recent studies on environmental or occupational health ${ }^{(2-10)}$ because the NOAEL has many shortcomings, e.g., not adequately reflecting the shape of the dose response and not appropriately accounting for study size. ${ }^{(11)}$ Nonetheless, since there is little practical experience in the BMD 
method (especially the hybrid method), ${ }^{(12)}$ many epidemiologists may arouse concern in applying the BMD method to clinical research: What is the relation between the values calculated from the BMD and NOAEL/LOAEL approaches? Or, did the BMD really correspond to the NOAEL or LOAEL?

The BMD is defined as the dose that corresponds to a specific change in an adverse response as compared to the response in unexposed subjects. ${ }^{(12)}$ It is determined by modeling a dose-response curve in the region of the dose-response relationship where biologically observable data are available. To take uncertainty of the data into consideration, the dose of interest is the lower $95 \%$ confidence limit (i.e., BMDL) on the BMD. On the other hand, the calculation of a NOAEL generally utilizes data that are categorized into distinct dose groups, and categorization of subjects into dose groups is an arbitrary process, ${ }^{(11)}$ while the BMD method does not rely on categorizations of either the response or the exposure. Such categorizations therefore may be problematic in most epidemiological studies. For instance, the risk for causing liver damage steadily increased when ethanol intake exceeded $30 \mathrm{~g} /$ day in the Dionysos study with categorized data, ${ }^{(13,14)}$ but the BMDL of ethanol intake for the aminotransferase abnormality was estimated to be approximately $50 \mathrm{~g} /$ day in Japanese salesmen of our study with the BMD method. ${ }^{(9)}$ The thresholds may have differed according to race, sorts of alcoholic beverage, or endpoints used.

The present article explores the relation between the BMDL and NOAEL (or LOAEL) in applying the BMD method to clinical research. As an example, we use data from a study carried out to investigate the effects of alcohol consumption on blood pressure in workers. Since subgroups who consumed an empirical range of ethanol (i.e., 0, 1-30, 31-60, 61-90, or $>90 \mathrm{~g}$ /day) have been utilized in the preceding alcohol research, ${ }^{(13,14)}$ we employ this categorization, together with the BMD method formulated by BudtzJørgensen et al. ${ }^{(6)}$

\section{MATERIALS AND METHODS}

\subsection{Study Subjects}

A self-reported questionnaire with detailed explanations of the study purpose was distributed to approximately 3,400 salesmen at motor vehicle dealerships in Akita, northeast Japan in 2002, and 1,244 of them consented to our proposal and returned the form to an occupational health nurse of a health insurance union (participation rate, 37\%). Also, 144 of the respondents were excluded according to the following criteria: subjects must have undergone the mandatory health checkup, conducted under the Industrial Safety and Health Law in Japan; they must not suffer from ischemic heart disease, chronic renal failure, alcoholic dependency diagnosed by the DSMIV ${ }^{(15)}$ liver cirrhosis, or liver cancer, or have been taking antihypertensive medication; also, the questionnaire had perfect data regarding drinking habit, etc. The study population, accordingly, consisted of 1,100 healthy men (respondents) aged 18-29 years (33.9\%), 30-39 years (23.1\%), 40-49 years $(24.6 \%)$, $50-59$ years $(16.9 \%)$, and $60-68$ years $(1.5 \%)$ and of 1,726 anonymous salesmen (i.e., nonrespondents) who underwent the above health checkup. The study protocol was approved by the ethical review committee of the Akita University School of Medicine.

\subsection{Data Collection}

The weekly amount of each type of alcoholic beverage consumed was asked as described previously; ${ }^{(9)}$ e.g., "How many $180-\mathrm{mL}$ cups (or 1,800-mL bottles) of sake do you usually drink in a week?" and "How many $350-\mathrm{mL}$ cans (or, $500-\mathrm{mL}$ cans or $633-\mathrm{mL}$ bottles) of beer do you usually drink in a week?" Types of alcoholic beverages listed were sake, beer, shochu (Japanese distilled alcoholic beverage primarily made from wheat or sweet potatoes), whisky, wine, and others (e.g., plum wine, brandy, gin, and vodka). A total of $100 \%$ ethanol equivalent dose (g/day) was calculated for each subject on the assumption that sake, beer, shochu, whisky, and wine contain $15 \%, 5 \%, 20$ (or 25 ) $\%, 40 \%$, and $12 \%$ of ethanol, respectively. Approximately $5 \%$ of 883 drinkers, who consumed $100 \%$ ethanol equivalent dose of more than $800 \mathrm{~g} /$ week, were directly asked about recent alcohol consumption by the occupational health nurse 5 months after the questionnaire collection in order to validate the reported alcohol consumption.

Data of systolic and diastolic blood pressure (SBP and DBP), together with serum total cholesterol (T-CH), HDL-cholesterol (HDL-CH), triglycerides (TG), and body mass index (BMI), were obtained from each record of the health checkup. Blood pressure was measured twice by a trained nurse with a mercury sphygmomanometer, 2 minutes after sitting in a chair with their backs supported and their arms bared and supported at heart level. The average of 
two measurements was used in the analysis. SBP and DBP were defined as the first and the fifth Korotkoff sounds. Subjects were asked not to smoke or to consume coffee or tea for 12 hours before the health checkup. Serum T-CH, HDL-CH, and TG were determined calorimetrically with enzymatic methods by the Akita Prefectural Center of Health Care (reference intervals were 130 to $220 \mathrm{mg} / \mathrm{dL}$ for T-CH, 41$70 \mathrm{mg} / \mathrm{dL}$ for HDL-CH, and $42-150 \mathrm{mg} / \mathrm{dL}$ for TG). Job stress and smoking habit were also examined in the questionnaire.

\subsection{Statistical Methods}

The comparisons in the data collected between the respondents and nonrespondents and between the respondents with and without hypertension were done by unpaired $t$ test or Mann-Whitney $U$ test (or $\chi^{2}$ test for comparison of proportions). Hypertension was conveniently defined as SBP $\geq 140 \mathrm{mmHg}$ or DBP $\geq 90 \mathrm{mmHg}$. The difference between daily ethanol intakes answered in the questionnaire and interview was analyzed by the paired-sample $t$ test; also, the relationship between daily ethanol consumption and HDL-CH was examined by the Spearman rank correlation coefficient $\left(r_{s}\right)$. The differences in SBP and DBP among categorized groups were compared using the two-way analysis of variance. The relations of daily ethanol intake and six possible confounders (age, BMI, log-transformed T-CH and TG, job stress, and smoking status) to SBP and DBP in the respondents were tested by multiple regression analysis. Multiple logistic regression analysis was used to calculate the odds ratio of daily ethanol intake to hypertension after controlling for the above confounders. The response to the question on job stress (i.e., "How stressed are you in your work life?") was scored as "not at all" = 1, "slightly" = 2, "moderately" $=3$, "very" $=4$, and "extremely" $=5$; also, the response to smoking status was scored as "nonsmoker or ex-smoker" $=0$ and "smoker" $=1$.

The BMD is defined as the daily ethanol dose that results in an increased probability of abnormal blood pressure by a benchmark response (BMR), i.e., from $P_{0}$ to $P_{0}+\mathrm{BMR}$ at the BMD, where the $P_{0}$ and BMR represent an abnormal probability of blood pressure in the nondrinking population and an excess risk in the drinking population, respectively (Fig. 1)..$^{(2,6,11)}$ Our statistical dose-effect model is decided on the basis of power functions $\left[g(d)=d^{K}\right]$, for the dependence of blood pressure on daily ethanol intake $(d)$, age,

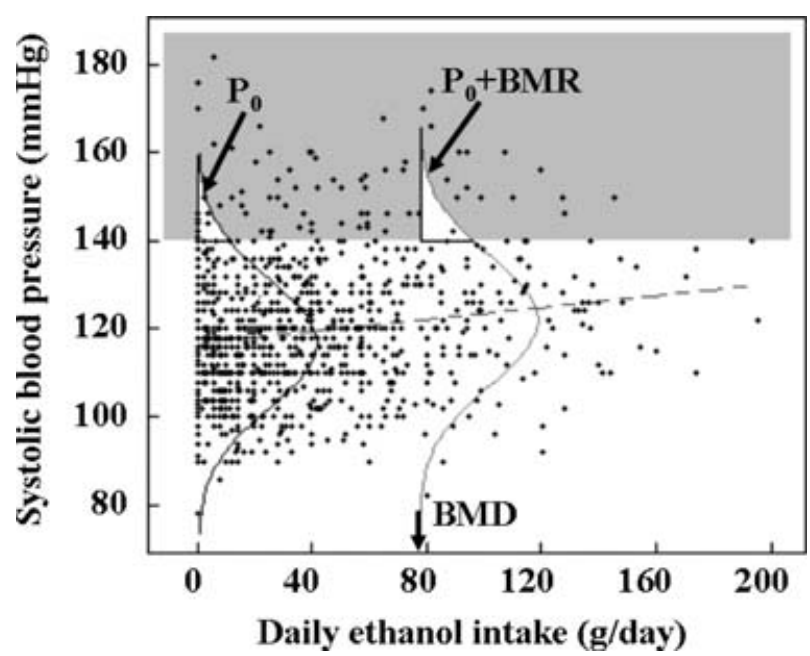

Fig. 1. Dose-response relationships between daily ethanol intake and systolic blood pressure in 1,100 Japanese salesmen for BMD calculation after adjusting for six confounders. The $P_{0}$, benchmark response (BMR), and BMD indicate an abnormal probability of blood pressure in the nondrinkers, an excess risk above $P_{0}$ in the drinkers, and benchmark dose, respectively (i.e., $P_{0}=0.05$ and $\mathrm{BMR}=0.05)$.

BMI, log-transformed T-CH and TG, job stress, and smoking status:

$$
\begin{aligned}
\mu(d)= & \beta_{0}+\beta g(d)+\beta_{1}(\text { age })+\beta_{2}(\mathrm{BMI}) \\
& +\beta_{3}[\log (\mathrm{T}-\mathrm{CH})]+\beta_{4}[\log (\mathrm{TG})] \\
& +\beta_{5}(\text { job stress })+\beta_{6}(\text { smoking status }),
\end{aligned}
$$

where $\mu(d)$ is the expected response of the subject. The power parameter $K$ has been restricted to values equal to 1 or over, thus allowing the dose-effect curve to be nonlinear. This BMD method is a hybrid approach and needs an abnormal response level to interpret continuous data of the endpoint. The cutoff value $(C)$ is defined so that the risk in an unexposed subject is $P_{0}$, and it depends on the above confounders. ${ }^{(6)}$ The cutoff value in subjects with all confounder values equal to 0 can be calculated with use of the following equation: $P_{0}=1-\Phi\left[\left(C-\beta_{0}\right) / \sigma\right]$, where $\Phi$ and $\sigma$ indicate the normal cumulative distribution function and the standard deviation $(S D)$ of blood pressure in the nondrinking population, respectively. In this study, as six confounders were included, the cutoff value for an average subject was computed by using the normalized value (i.e., $\left[X_{i}-X_{\text {mean }}\right] /$ $S D)$ for each confounder $\left(X_{i}\right)$. Then, the BMD was given by $\mathrm{BMD}=g^{-1}\left\{\left[\Phi^{-1}\left(1-P_{0}\right)-\Phi^{-1}\left(1-P_{0}-\right.\right.\right.$ $\mathrm{BMR})] \sigma / \beta\}$, and the BMDL was calculated as the 


\begin{tabular}{|c|c|c|c|c|}
\hline & \multicolumn{2}{|c|}{ 1,100 Respondents } & \multicolumn{2}{|c|}{ 1,726 Nonrespondents } \\
\hline & Mean $(S D)$ & Range & Mean $(S D)$ & Range \\
\hline Age (years) & $37.3(11.4)$ & $18-68$ & $35.7(11.1)$ & $18-66$ \\
\hline Body mass index $\left(\mathrm{kg} / \mathrm{m}^{2}\right)$ & $23.2(3.4)$ & $14.5-43.8$ & $23.1(3.3)$ & $15.9-40.8$ \\
\hline Total cholesterol (mg/dL) & $195^{*}$ & $106-317$ & $190^{*}$ & $109-371$ \\
\hline Triglycerides (mg/dL) & $93^{*}$ & $15-1252$ & $88^{*}$ & $19-1442$ \\
\hline Systolic blood pressure $(\mathrm{mmHg})$ & $119(15)$ & $78-182$ & $118(14)$ & 71-190 \\
\hline Diastolic blood pressure $(\mathrm{mmHg})$ & $73(12)$ & $40-118$ & $73(12)$ & $40-118$ \\
\hline \multicolumn{5}{|l|}{ 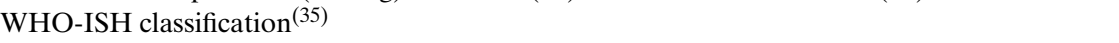 } \\
\hline Normal blood pressure (\%) & 80.1 & & 76.0 & \\
\hline High-normal blood pressure (\%) & 6.7 & & 11.4 & \\
\hline Hypertension (\%) & 13.2 & & 12.6 & \\
\hline
\end{tabular}

Table I. Basal Characteristics of Japanese Salesmen

*Median value.

lower $95 \%$ confidence limit of the BMD. ${ }^{(6)}$ We used $P_{0}$ values of 0.05 and 0.1 and BMR values of $0.02,0.05$, and 0.1. All analyses, with two-sided $p$ values, were performed by using the Statistical Package for the Biosciences (SPBS V9.51) with the BMD program. ${ }^{(16)}$

\section{RESULTS}

The basal characteristics in the 1,100 respondents and 1,726 nonrespondents are shown in Table I. There was only a significant difference in age between the two groups. In 45 respondents who consumed 100\% ethanol equivalent dose of more than $800 \mathrm{~g} /$ week, the daily ethanol intake answered in the questionnaire did not differ from that in the interview $(p>0.2$; data not shown). Also, the daily ethanol intake in the respondents was significantly correlated with the HDL-CH $\left(r_{s}=0.155 ; p<0.001\right)$. Proportions of mainly beer and shochu consumers among the respondents were $48.6 \%$ and $19.5 \%$, respectively.
In all respondents, daily ethanol intake, BMI, $\mathrm{T}-\mathrm{CH}$, TG, and the proportion of drinkers were significantly higher in the hypertensives than in the normotensives (Table II), while the smoking status was significantly lower in the hypertensives. Also, the SBP and DBP tended to be elevated according to aging and increased daily ethanol intake (Table III). The daily ethanol intake was significantly related to SBP and DBP (Table IV) when adjusting for confounders such as age, BMI, and smoking status. The risk for hypertension (i.e., $\mathrm{SBP} \geq 140 \mathrm{mmHg}$ or $\mathrm{DBP} \geq 90 \mathrm{mmHg}$ ) increased significantly when daily ethanol intake exceeded $60 \mathrm{~g} /$ day (Table $\mathrm{V}$ ); that is, the NOAEL of daily ethanol intake was thought to be $60 \mathrm{~g} /$ day, and the LOAEL was estimated to lie somewhere between 60.1 and $90.0 \mathrm{~g} /$ day.

When the $K$-power and linear dose-response models were applied (Table VI), the BMDLs of daily ethanol intake, adjusted by six confounders and set at the $P_{0}$ of 0.05 and the BMR of 0.05 , were $60 \mathrm{~g} /$ day

\begin{tabular}{|c|c|c|c|c|c|}
\hline & \multicolumn{2}{|c|}{ Without Hypertension } & \multicolumn{2}{|c|}{ With Hypertension } & \multirow[b]{2}{*}{$p$-Value } \\
\hline & Mean & $S D$ & Mean & $S D$ & \\
\hline Age (years) & 36.3 & 11.1 & 43.8 & 10.8 & $<0.0001$ \\
\hline Body mass index $\left(\mathrm{kg} / \mathrm{m}^{2}\right)$ & 22.8 & 3.2 & 25.2 & 3.9 & $<0.0001$ \\
\hline Total cholesterol (mg/dL) & $191^{*}$ & 1.20 & $204^{*}$ & 1.19 & $<0.0001$ \\
\hline HDL-cholesterol (mg/dL) & 57 & 14 & 57 & 14 & 0.8937 \\
\hline Triglycerides (mg/dL) & $93^{*}$ & 1.91 & $124^{*}$ & 1.87 & $<0.0001$ \\
\hline Systolic blood pressure (mmHg) & 115 & 11 & 143 & 13 & $<0.0001$ \\
\hline Diastolic blood pressure $(\mathrm{mmHg})$ & 71 & 9 & 92 & 9 & $<0.0001$ \\
\hline Job stress (score) & 2.2 & 1.0 & 2.3 & 1.0 & 0.3892 \\
\hline Smokers (\%) & 70.9 & & 53.8 & & $<0.0001$ \\
\hline Drinkers (\%) & 79.2 & & 87.6 & & 0.0175 \\
\hline Daily ethanol intake (g/day) & 30 & 34 & 48 & 30 & $<0.0001$ \\
\hline
\end{tabular}

Table II. Differences in Main Characteristics Between 955 Respondents Without Hypertension and 145 Respondents with Hypertension in Japan

${ }^{*}$ Geometric mean. 
Table III. Systolic and Diastolic Blood Pressure (SBP and DBP) in Categorized Groups of Japanese Salesmen by Age and Daily Ethanol Intake (Number, Mean, and Standard Deviation in Parentheses)

\begin{tabular}{|c|c|c|c|c|c|c|c|c|c|c|c|c|}
\hline \multirow[b]{2}{*}{ Ethanol Intake (g/day) } & \multicolumn{3}{|c|}{ Under 29 Years } & \multicolumn{3}{|c|}{ 30-39 Years } & \multicolumn{3}{|c|}{ 40-49 Years } & \multicolumn{3}{|c|}{ Above 50 Years } \\
\hline & $N$ & SBP & DBP & $N$ & SBP & DBP & $N$ & SBP & DBP & $N$ & SBP & $\mathrm{DBP}$ \\
\hline Nondrinkers & 111 & $113(13)$ & $66(11)$ & 41 & $122(15)$ & $76(12)$ & 35 & $115(12)$ & $72(9)$ & 30 & $115(15)$ & $69(9)$ \\
\hline $0.1-30.0$ & 180 & $114(13)$ & $67(9)$ & 114 & $117(12)$ & $72(10)$ & 93 & $119(14)$ & $77(11)$ & 62 & $122(16)$ & $76(10)$ \\
\hline $30.1-60.0$ & 47 & $116(11)$ & $69(10)$ & 57 & $118(11)$ & $75(9)$ & 71 & $123(16)$ & $80(12)$ & 58 & $125(16)$ & $80(10)$ \\
\hline $60.1-90.0$ & 19 & $114(12)$ & $68(12)$ & 19 & $123(15)$ & $72(12)$ & 42 & $122(15)$ & $79(10)$ & 30 & $130(22)$ & $80(14)$ \\
\hline$>90.0$ & 16 & $121(13)$ & $75(12)$ & 23 & $127(14)$ & $80(13)$ & 30 & $128(14)$ & $83(11)$ & 22 & $127(17)$ & $78(11)$ \\
\hline
\end{tabular}

Note: Two-way analysis of variance, $p<0.0001$.

for SBP and $58 \mathrm{~g} /$ day for DBP. In the same table, the BMDs and BMDLs calculated from data without any confounders were somewhat smaller than those after adjusting for six confounders.

\section{DISCUSSION}

There are a large number of reports on the effects of chronic ethanol intake on blood pressure, and ethanol has been established as an exacerbating factor of hypertension, preceding coronary heart disease or stroke. ${ }^{(17)}$ At the same time, several issues to be solved in such studies also have been raised, e.g., Was the estimation of daily ethanol intake appropriate? For that reason, we should first consider our results before discussing application of the BMD method to clinical research.

\subsection{Effects of Daily Ethanol Intake on Blood Pressure}

In this study, we observed significant doseresponse relationships between the daily ethanol intake and both SBP and DBP in the Japanese sales-

Table IV. Relations of Daily Ethanol Intake and Confounders to Systolic and Diastolic Blood Pressures in 1,100 Japanese Salesmen (Regression Coefficient and $p$-Value in Parentheses)

\begin{tabular}{lcc}
\hline & $\begin{array}{c}\text { Systolic } \\
\text { Blood Pressure }\end{array}$ & $\begin{array}{c}\text { Diastolic } \\
\text { Blood Pressure }\end{array}$ \\
\hline Daily ethanol intake & $0.0677(<0.0001)$ & $0.0560(<0.0001)$ \\
Age & $0.2066(<0.0001)$ & $0.2836(<0.0001)$ \\
Body mass index & $1.1383(<0.0001)$ & $0.8228(<0.0001)$ \\
Log[T-CH] & $2.0409(0.7329)$ & $3.0948(0.5032)$ \\
Log[TG] & $2.8566(0.0885)$ & $2.1431(0.0983)$ \\
Job stress & $0.5888(0.1351)$ & $0.1731(0.5696)$ \\
Smoking status & $-3.2609(0.0003)$ & $-2.1675(0.0020)$ \\
\hline
\end{tabular}

Note: Results of multiple regression analysis. men (Tables III and IV). As shown in Fig. 1 and Table III, the linear model appears to be more appropriate than a J-shaped or U-shaped model. ${ }^{(18,19)}$ Also, other Japanese studies reported independent linear associations of alcohol with blood pressure. ${ }^{(20,21)}$ This may have been due to the fact that Japanese people, including our subjects, generally prefer beer and shochu to wine, while some German studies have shown a J-shaped association between alcohol and blood pressure. ${ }^{(17,22)}$

The multiple logistic regression analysis using categorical data showed that the increased ethanol intake was significantly linked with hypertension, and it indicated that the odds ratio for hypertension was statistically significant when daily ethanol intake exceeded $60 \mathrm{~g} /$ day (Table V). Nonetheless, this lies within the upper limit of moderate alcohol consumption ranging from 24 to $80 \mathrm{~g} /$ day of $100 \%$ ethanol; ${ }^{(17)}$ it is considerably higher than other thresholds of alcohol causing hypertension, which have been reported

Table V. Relations of Daily Ethanol Intake and Confounders to Hypertension (SBP $\geq 140 \mathrm{mmHg}$ or DBP $\geq 90 \mathrm{mmHg}$ ) in 1,100 Salesmen

$95 \%$

\begin{tabular}{lcc}
\hline & & $\begin{array}{c}95 \% \\
\end{array}$ \\
& Odds Ratio & Confidence Limit \\
\hline $\begin{array}{l}\text { Daily ethanol intake (g/day) } \\
\quad \text { Nondrinkers }\end{array}$ & \\
$0.1-30.0$ & 1.000 (reference) & \\
30.1-60.0 & 1.252 & $0.667,2.347$ \\
$60.1-90.0$ & 1.885 & $0.975,3.647$ \\
$>90.0$ & 2.877 & $1.377,6.010$ \\
Age (years) & 4.109 & $1.962,8.606$ \\
Body mass index (kg/m $\left.{ }^{2}\right)$ & 1.058 & $1.037,1.079$ \\
Log[T-CH (mg/dL)] & 1.257 & $1.181,1.338$ \\
Log[TG (mg/dL)] & 0.423 & $0.023,7.669$ \\
Job stress (score) & 1.319 & $0.612,2.839$ \\
Smoking status & 1.040 & $0.866,1.250$ \\
\hline
\end{tabular}

Note: Results of multiple logistic regression analysis. 
Table VI. BMDs and BMDLs of Daily Ethanol Intake (g/day) for Systolic and Diastolic Blood Pressures (SBP and DBP) in 1,100 Salesmen

\begin{tabular}{|c|c|c|c|c|c|c|c|c|c|c|c|c|}
\hline & \multicolumn{6}{|c|}{$K$-Power Model } & \multicolumn{6}{|c|}{ Linear Model $(K=1)$} \\
\hline & \multicolumn{2}{|c|}{$\mathrm{BMR}=0.02$} & \multicolumn{2}{|c|}{$\mathrm{BMR}=0.05$} & \multicolumn{2}{|c|}{$\mathrm{BMR}=0.10$} & \multicolumn{2}{|c|}{$\mathrm{BMR}=0.02$} & \multicolumn{2}{|c|}{$\mathrm{BMR}=0.05$} & \multicolumn{2}{|c|}{$\mathrm{BMR}=0.10$} \\
\hline & BMD & BMDL & BMD & BMDL & BMD & BMDL & BMD & BMDL & $\mathrm{BMD}$ & BMDL & BMD & BMDL \\
\hline \multicolumn{13}{|c|}{ After adjusting for six confounders } \\
\hline \multicolumn{13}{|c|}{$P_{0}=0.05^{\mathrm{a}}$} \\
\hline SBP & 36 & 28 & 78 & 60 & 129 & 100 & 36 & 28 & 77 & 60 & 129 & 100 \\
\hline DBP & 34 & 27 & 74 & 58 & 123 & 97 & 34 & 27 & 74 & 58 & 123 & 97 \\
\hline \multicolumn{13}{|c|}{$P_{0}=0.10^{\mathrm{a}}$} \\
\hline SBP & 23 & 18 & 53 & 41 & 94 & 72 & 23 & 17 & 52 & 40 & 93 & 72 \\
\hline DBP & 22 & 17 & 50 & 39 & 90 & 70 & 22 & 17 & 50 & 39 & 90 & 70 \\
\hline \multicolumn{13}{|c|}{ Crude data (not including any confounders) } \\
\hline \multicolumn{13}{|c|}{$P_{0}=0.05^{\mathrm{a}}$} \\
\hline SBP & 28 & 22 & 59 & 48 & 98 & 80 & 27 & 22 & 58 & 47 & 98 & 79 \\
\hline DBP & 23 & 19 & 49 & 41 & 83 & 69 & 23 & 19 & 49 & 41 & 83 & 69 \\
\hline \multicolumn{13}{|c|}{$P_{0}=0.10^{\mathrm{a}}$} \\
\hline SBP & 17 & 14 & 40 & 32 & 71 & 58 & 17 & 14 & 39 & 32 & 71 & 57 \\
\hline DBP & 15 & 12 & 33 & 28 & 60 & 50 & 15 & 12 & 33 & 28 & 60 & 50 \\
\hline
\end{tabular}

${ }^{a} P_{0}$ and BMR indicate an abnormal probability of blood pressure in the nondrinkers and an excess risk in the drinkers, respectively. Note: Cutoff values calculated from the BMD models, after adjusting for six confounders (when confounder was not used in parentheses), were $140(140) \mathrm{mmHg}$ for SBP and 90 (89) $\mathrm{mmHg}$ when $P_{0}=0.05$; and 135 (134) $\mathrm{mmHg}$ for SBP and 86 (85) $\mathrm{mmHg}$ for DBP when $P_{0}=0.10$.

to be $30 \mathrm{~g}$ /day in Brazilian and North American populations $^{(18,23)}$ and less than $23 \mathrm{~g} /$ day in southwestern Japan. ${ }^{(24)}$ Four possible explanations for this disagreement are as follows:

1. The thresholds may be different between the general population and workers because of the healthy worker's effect.

2. Mean age in the study population may have differed among those researches, even if each study adjusted for age. Since blood pressure, as well as the prevalence of hypertension, increases with aging (Table III), the elderly population may be more susceptible to alcohol than the middle-aged population.

3. The regional difference in alcohol/aldehyde dehydrogenase (ADH/ALDH) genotypes may have affected the outcomes because there exists a highly prevalent polymorphism in the low $\mathrm{Km} \mathrm{ALDH}(\mathrm{ALDH} 2)$ gene in Japanese as compared with the Caucasoid; (25) also, the distribution of such genotypes is greatly different within Japan.

4. The study designs, i.e., a cross-sectional and cohort studies, were different.

Concerning the self-reported questionnaire used for the exposure assessment, the salesmen of our study were asked about the number of cans (or bottles) of each alcoholic beverage per week concretely, ${ }^{(9)}$ although a questionnaire utilized in many health examination centers of Japan included a cognitively difficult task to summarize drinking behavior, ${ }^{(17)}$ e.g., subjects have to calculate the equivalent dose of sake per day if they drink beer or whisky. ${ }^{(26)}$ A significant association was observed between the daily ethanol intake and HDL-CH in the 1,100 salesmen; by showing the above correlation, qualitative evidence is provided that alcohol use is being measured with at least some degree of validity. ${ }^{(17,27)}$ Also, the daily ethanol intake was confirmed in 45 salesmen who drank too much by interview, to avoid inaccuracy due to underreporting of alcohol consumption. ${ }^{(17,18,28)}$ In addition, there were positively linear relationships between weekly ethanol intake and liver enzyme activities in the same salesmen. ${ }^{(9)}$ Thus, our questionnaire seemed to provide an appropriate estimate of daily ethanol intake.

In this study, there may have been possible limitations, e.g., sampling, selection, or confounding biases. However, proportions of $31,800,000$ male employees in Japan in 2001 were $23.4 \%$ at $18-29$ years of age, $23.7 \%$ at $30-39$ years, $21.3 \%$ at $40-49$ years, $23.1 \%$ at $50-59$ years, and $8.4 \%$ at $60-69$ years, ${ }^{(29)}$ and were similar to those of our study population regarding 
age distribution. Also, since annual alcohol consumption per household in Akita has been reported to be more than that in other Japanese prefectures, ${ }^{(30)}$ the statistical power may have been potent because of a wide range of alcohol intake. The participation rate was not so high, but the characteristics did not differ between the respondents and nonrespondents in this study population (Table I). In addition, possible confounders such as age, BMI, job stress, and smoking status, except for physical activity and sodium intake, were considered in the process of data analysis. Therefore, it is suggested that our findings were not heavily influenced by such biases.

This study indicated that smoking status affected blood pressure levels negatively (Table IV). Some epidemiologic studies also have reported that SBP and DBP were lower in smokers than in nonsmokers. ${ }^{(31)}$ This would be regarded as a paradox because nicotine has potent sympathomimetic effects, affecting blood pressure levels and heart rate. ${ }^{(32)}$ On the other hand, blood pressure was not affected by T-CH, TG, or job stress in the regression analysis of this study. The associations between job stress and hypertension remain disputable. ${ }^{(33)}$ Further research with other new scales is necessary to reconfirm such effects of smoking status and job stress on blood pressure.

\subsection{Application of BMD Approach to Clinical Research}

In the BMD approach that was set at the $P_{0}$ of 0.05 and BMR of 0.05 , the BMDL of daily ethanol intake for increased blood pressure was perfectly accordant with the NOAEL for hypertension diagnosed by $\mathrm{SBP} \geq 140 \mathrm{mmHg}$ or $\mathrm{DBP} \geq 90 \mathrm{mmHg}$, while two approaches were mathematically independent. In addition, the calculated BMD was between 60.1 and $90.0 \mathrm{~g} /$ day in the groups categorized (Table V). Despite the uncertainties of the data used, therefore, these findings suggest that the BMDL and BMD correspond to the NOAEL and LOAEL, respectively. On the other hand, it is known that the BMD approach reflects sample size more appropriately than a NOAEL, and smaller studies tend to result in smaller BMDLs, whereas the opposite is true for NOAELs. ${ }^{(11,12)}$ If the sample number of clinical data be smaller, the true threshold would be placed somewhere between the BMDL and BMD calculated from the research.

All BMDs and BMDLs calculated from data without any confounders were smaller than those calculated from data with six confounders (Table VI). Blood pressure was shown to depend on age, BMI, and smoking status, as well as alcohol (Table IV), and the regression coefficients $(\beta)$ of daily ethanol intake to SBP and DBP, when no confounders were included, were approximately 10 times larger than those when six confounders were employed. This would be due to the fact that there were a large number of nondrinking salesmen at ages of less than 30 years (Table III), indicating that the exposure effect was reduced after confounder adjustment. On the other hand, BudtzJørgensen et al. have described that a higher BMDL is obtained if a strong predictor of the response that is not related to the exposure is excluded from the set of independent variables. ${ }^{(6)}$ In either case, important confounders should be considered in epidemiological research.

In the BMD method, the abnormal response level to interpret continuous data of the endpoint can be specified by defining either a cutoff value $(C)$ or the unexposed risk $\left(P_{0}\right)$, and these values depend on each other. When we used the $P_{0}$ of 0.05 , the cutoff values calculated by our method, i.e., $140 \mathrm{mmHg}$ for SBP and $90 \mathrm{mmHg}$ for DBP (Table VI), were consistent with the upper limits of the "high-normal" or "prehypertension" classification by the WHO-ISH ${ }^{(34)}$ or JNC VII. ${ }^{(35)}$ Thus, this approach seems to be preferred in terms of statistical efficiency and statistical validity as compared to transformation of data into dichotomous format. ${ }^{(12,36)}$ Also, it is crucial to confirm the difference between the cutoff value computed and the clinical standard value (e.g., the upper normal limit of an endpoint) in deciding which dose-response model to use for a clinical data set because such cutoff values could be changed by different confounder values used, ${ }^{(6)}$ especially mean age in each study.

The BMR refers to a specific risk increase above background risk in BMD calculations for continuous outcomes. ${ }^{(2)}$ In the quantal-response setting, BMR values of $0.1,0.05$, or possibly 0.01 are generally chosen, though the EPA water quality criteria guidelines recommend using either BMR $=0.05$ or $0.1 .^{(37)}$ Also, any need for conservatism could be accommodated in the choice for the BMR. ${ }^{(11)}$ When $P_{0}=0.05$, the choice of $\mathrm{BMR}=0.05$ results in a BMD that represents a doubling of the proportion of the population that falls into the adverse effect region. Considering the consistency of thresholds computed in this study, the selected BMR value of 0.05 (and the $P_{0}$ of 0.05 ) appears to be more compatible with results of the multiple logistic regression analysis than the BMR of 0.02 or 0.1 (Table VI).

In epidemiological studies, the odds ratio and relative risk are frequently used, ${ }^{(18,21,38)}$ but such values 
depend on the interval or range used arbitrarily. For this reason, any changable threshold could be estimated from dose groups categorized. The BMD approach, contrarily, provides a promising method for calculating a threshold (point of departure) of the dose-response relation of risk factors in the study population given. ${ }^{(2,8,11)}$ In addition, the BMDs and BMDLs derived from a study on the effects of lead on $\delta$-aminolevulinic acid levels in plasma, blood, and urine of lead-exposed workers have been shown to be biologically valid. ${ }^{(8)}$ Although any BMD approaches should not be applied if there is no significant relationship between an exposure and outcome, the implication of this article is that the BMD method used in our study is very much available for researches in clinical medicine, as well as in environmental and occupational health and toxicology.

\section{ACKNOWLEDGMENTS}

We thank Drs. Esben Budtz-Jørgensen and Philippe Grandjean for their valuable advice on BMD calculations. This research was supported in part by the grants-in-aids for scientific research from the Nissan Science Foundation and from the Japan Ministry of Education, Culture, Sports and Technology.

\section{REFERENCES}

1. James, R. C., Warren, D. A., Halmes, C., \& Roberts, S. M. (2000). Risk assessment. In P. L. Williams, R. C. James, \& S. M. Roberts (Eds.), Principles of Toxicology: Environmental and Industrial Applications, 2nd ed. (pp. 437-477). New York: John Wiley.

2. National Research Council. (2000). Toxicological Effects of Methylmercury. Washington, DC: National Academy Press.

3. Haag-Grönlund, M., Fransson-Steen, R., \& Victorin, K. (1995). Application of the benchmark method to risk assessment of trichloroethene. Regular Toxicology and Pharmacology, 21, 261-269.

4. Crump, K. S., Kjellström, T., Shipp, A. M., Silvers, A., \& Stewart, A. (1998). Influence of prenatal mercury exposure upon scholastic and psychological test performance: Benchmark analysis of a New Zealand cohort. Risk Analysis, 18, 701-713.

5. Mutti, A., \& Smargiassi, A. (1998). Selective vulnerability of dopaminergic systems to industrial chemicals: Risk assessment of related neuroendocrine changes. Toxicology and Industrial Health, 14, 311-323.

6. Budtz-Jørgensen, E., Keiding, N., \& Grandjean, P. (2001). Benchmark dose calculation from epidemiological data. Biometrics, 57, 698-706.

7. Murata, K., Budtz-Jørgensen, E., \& Grandjean, P. (2002). Benchmark dose calculations for methylmercury-associated delays on evoked potential latencies in two cohorts of children. Risk Analysis, 22, 465-474.

8. Murata, K., Sakai, T., Morita, Y., Iwata, T., \& Dakeishi, M. (2003). Critical dose of lead affecting $\delta$-aminolevulinic acid levels. Journal of Occupational Health, 45, 209-214.
9. Dakeishi, M., Iwata, T., Ishii, N., \& Murata, K. (2004). Effects of alcohol consumption on hepatocellular injury in Japanese men. Tohoku Journal of Experimental Medicine, 202, 31-39.

10. Budtz-Jørgensen, E., Keiding, N., \& Grandjean, P. (2004). Effects of exposure imprecision on estimation of the benchmark dose. Risk Analysis, 24, 1689-1696.

11. Crump, K. (2002). Critical issues in benchmark calculations from continuous data. Critical Reviews in Toxicology, 32, 133153.

12. Filipsson, A. F., Sand, S., Nilsson, J., \& Victorin, K. (2003). The benchmark dose method-Review of available models, and recommendations for application in health risk assessment. Critical Reviews in Toxicology, 33, 505-542.

13. Bellentani, S., Saccoccio, G., Costa, G., Tiribelli, C., Manenti, F., Sodde, M., Saveria Croce, L., Sasso, F., Pozzato, G., Cristianini, G., Brandi, G., \& the Dionysos Study Group. (1997). Drinking habits as cofactors of risk for alcohol induced liver damage. Gut, 41, 845-850.

14. Bellentani, S., \& Tiribelli, C. (2001). The spectrum of liver disease in the general population: Lesson from the Dionysos study. Journal of Hepatology, 35, 531-537.

15. American Psychiatric Association. (1994). Diagnostic and Statistical Manual of Mental Disorders, 4th ed. Washington, DC: American Psychiatric Association.

16. Murata, K., \& Yano, E. (2002). Medical Statistics for EvidenceBased Medicine with SPBS User's Guide. Tokyo: Nankodo Publishers.

17. Grobbee, D. E., Rimm, E. B., Keil, U., \& Renaud, S. (1999). Alcohol and the cardiovascular system. In I. MacDonald (Ed.), Health Issues Related to Alcohol Consumption, 2nd ed. (pp. 125-179). Oxford: Blackwell Science.

18. Fuchs, F. D., Chambless, L. E., Whelton, P. K., Nieto, F. J., \& Heiss, G. (2001). Alcohol consumption and the incidence of hypertension: The atherosclerosis risk in communities study. Hypertension, 37, 1242-1250.

19. Murray, R. P., Connett, J. E., Tyas, S. L., Bond, R., Ekuma, O., Silversides, C. K., \& Barnes, G. E. (2002). Alcohol volume, drinking pattern, and cardiovascular disease morbidity and mortality: Is there a U-shaped function? American Journal of Epidemiology, 155, 242-248.

20. Ueshima, H., Shimamoto, T., Iida, M., Konishi, M., Tanigaki, M., Doi, M., Tsujioka, K., Nagano, E., Tsuda, C., Ozawa, H., Kojima, S., \& Komachi, Y. (1984). Alcohol intake and hypertension among urban and rural Japanese populations. Journal of Chronic Diseases, 37, 585-592.

21. Kondo, K., \& Ebihara, A. (1984). Alcohol consumption and blood pressure in a rural community of Japan. In W. Lovenberg \& Y. Yamori (Eds.), Nutritional Prevention of Cardiovascular Disease (pp. 217-224). Orlando: Academic Press.

22. Keil, U., Chambless, L., \& Remmers, A. (1989) Alcohol and blood pressure: Results from the Luebeck blood pressure study. Preventive Medicine, 18, 1-10.

23. Moreira, L. B., Fuchs, F. D., Moraes, R. S., Bredemeier, M., \& Duncan, B. B. (1998). Alcohol intake and blood pressure: The importance of time elapsed since last drink. Journal of Hypertension, 16, 175-180.

24. Ohmori, S., Kiyohara, Y., Kato, I., Kubo, M., Tanizaki, Y., Iwamoto, H., Nakayama, K., Abe, I., \& Fujishima, M. (2002). Alcohol intake and future incidence of hypertension in a general Japanese population: The Hisayama study. Alcoholism: Clinical and Experimental Research, 26, 1010-1016.

25. Goedde, H. W., Agarwal, D. P., Fritze, G., Meier-Tackmann, D., Singh, S., Beckmann, G., Bhatia, K., Chen, L. Z., Fang, B., Lisker, R., Paik, Y. K., Rothhammer, F., Saha, N., Segal, B., Srivastava, L. M., \& Czeizel, A. (1992). Distribution of ADH2 and ALDH2 genotypes in different populations. Human Genetics, 88, 344-346.

26. Nagaya, T., Yoshida, H., Takahashi, H., Matsuda, Y., \& Kawai, M. (1999). Dose-response relationships between drinking and 
serum tests in Japanese men aged 40-59 years. Alcohol, 17, 133-138.

27. Giovannucci, E., Colditz, G., Stampfer, M. J., Rimm, E. B., Litin, L., Sampson, L., \& Willett, W. C. (1991). The assessment of alcohol consumption by a simple self-administered questionnaire. American Journal of Epidemiology, 133, 810817.

28. Steffensen, F. H., Sørensen, H. T., Brock, A., Vilstrup, H., \& Lauritzen, T. (1997). Alcohol consumption and serum liverderived enzymes in a Danish population aged 30-50 years. International Journal of Epidemiology, 26, 92-99.

29. Ministry of Public Management, Home Affairs, Posts and Telecommunications. (2002). The Annual Report on the Labour Force Survey 2001. Tokyo: Statistics Bureau, Ministry of Public Management, Home Affairs, Posts and Telecommunications.

30. Ministry of Public Management, Home Affairs, Posts and Telecommunications. (2002). The Annual Report on the Family Income and Expenditure Survey 2001. Tokyo: Statistics Bureau, Ministry of Public Management, Home Affairs, Posts and Telecommunications.

31. Green, M. S., Jucha, E., \& Luz, Y. (1986). Blood pressure in smokers and nonsmokers: Epidemiologic findings. American Heart Journal, 111, 932-940.

32. U. S. Surgeon General. (1988). Reducing the Health Consequences of Smoking: Nicotine Addiction. Washington, DC: U.S. Department of Health and Human Services.

33. Schnall, P. L., \& Landsbergis, P. A. (1994). Job strain and cardiovascular disease. Annual Reviews in Public Health, 15, 381-411.

34. WHO-ISH Guidelines Subcommittee. (1999). 1999 World Health Organization-International Society of Hypertension guidelines for the management of hypertension. Journal of Hypertension, 17, 151-183.

35. Chobanian, A. V., Bakris, G. L., Black, H. R., Cushman, W. C., Green, L. A., Izzo, J. L., Jr., Jones, D. W., Materson, B. J., Oparil, S., Wright, J. T., Jr., \& Roccella, E. J., Joint National Committee on Prevention, Detection, Evaluation, and Treatment of High Blood Pressure, National Heart, Lung, and Blood Institute, \& National High Blood Pressure Education Program Coordinating Committee. (2003). Seventh report of the Joint National Committee on prevention, detection, evaluation, and treatment of high blood pressure. Hypertension, $42,1206-1252$.

36. West, R., \& Kodell, R. (1999). A comparison of methods of benchmark-dose estimation for continuous response data. Risk Analysis, 19, 453-459.

37. U.S. EPA. (2000). Methodology for Deriving Ambient Water Quality Criteria for the Protection of Human Health. Technical Support Document Volume 1: Risk Assessment (EPA-822-B00-005). Washington, DC: Office of Water, Office of Science and Technology, U.S. Environmental Protection Agency.

38. Klag, M. J., Moore, R. D., Whelton, P. K., Sakai, Y., \& Comstock, G. W. (1990). Alcohol consumption and blood pressure: A comparison of native Japanese to American men. Journal of Clinical Epidemiology, 43, 1407-1414. 